\title{
Intestinibacter bartlettii
}

National Cancer Institute

\section{Source}

National Cancer Institute. Intestinibacter bartlettii. NCI Thesaurus. Code C122324.

A species of obligately anaerobic, Gram-positive, rod-shaped, non-motile, spore-forming bacteria in the family Clostridiaceae. I. bartlettii is neg ative for lecithinase, lipase, catalase and urease, and is unable to hydrolyze esculin or gelatin, or reduce nitrate to nitrite, but positive for acid phosphatase, galactosidase and beta-glucuronidase. The species was originally isolated from fecal samples. 